\title{
The Relationship between the Serum Level of Selenium and Cervical Intraepithelial Neoplasia: A Comparative Study in a Population of Nigerian Women
}

\author{
Ehiozogie Obhielo ${ }^{1}$, Michael Ezeanochie ${ }^{2 *}$, Oghenefegor Olokor $\mathrm{O}^{2}$, Anthony \\ Okonkwo $^{2}$, Etedafe Gharoro ${ }^{2}$
}

\begin{abstract}
Objective: To determine the relationship between the serum level of selenium and cervical intraepithelial neoplasia (CIN). Methods: A case controlled study that compared the serum level of selenium in 45 women with CIN (cases) to 45 women (age matched controls) with normal cervical cytology. Socio-demographic data and information on known risk factors for cervical cancer among the sample was compared between both groups using inferential statistics. Results: There was no significant difference in the mean selenium values between the cases and controls [p- 0.076 , 95\% CI (-15.08 - 0.76)]. However, subgroup analysis showed a statistically significant difference between patients with normal cervical cytology, CIN I, II and III ( $p=0.021)$. In addition, there was also significant difference in the selenium level between women with normal cervical cytology and CIN III ( $p$ value $=0.016$ ) with a significant inverse linear trend $(\mathrm{p}=0.025)$. Conclusion: With increasing severity of $\mathrm{CIN}$, a significant reduction in the level of selenium in serum was observed. This reducing value of serum selenium, a surrogate marker for increased oxidative stress, may be important factor for the development of persistent HPV infection and in particular high grade CIN III lesions. This observation requires further research.
\end{abstract}

Keywords: Selenium- cervical intraepithelial neoplasia- cancer- Nigeria

Asian Pac J Cancer Prev, 20 (5), 1433-1436

\section{Introduction}

Cervical cancer, with an estimated 528,000 new cases and 266,000 deaths annually, is a leading cause of death from cancer among women worldwide especially in developing countries (Franco et al., 2003; Ferlay et al., 2015). Oncogenic human papilloma virus (HPV) infection has been established as a 'necessary' but not solely sufficient cause of cervical cancer. About $80 \%$ of sexually active individuals become infected with a high-risk HPV type with $90 \%$ of these infections cleared by immune responses of the host within two years (Ma et al., 2017). The exact determinants and mechanisms modulating viral clearance or persistence (cofactors) are not quite clear. Therefore, additional research focused on identifying these additional HPV cofactors for better cervical cancer control has been advocated (Franceschi et al., 2015; Muwonge et al., 2016; Chih et al., 2013).

Several studies have linked dietary factors, to cervical cancers either as promoters or inhibitors of carcinogenesis (Ozgu et al., 2016; Jia et al., 2016; Cunzhi et al., 2003; Kim et al., 2003). Selenium an essential trace element and component of the intracellular antioxidant glutathione peroxidise enzyme has been shown from several lines of evidence to have a protective effect against cancer development (Cunzhi et al., 2003; Kim et al., 2003). Recent studies suggest that risk of premalignant disease of the cervix increases with decreasing serum antioxidant system activity and reduced serum levels of micronutrients such as selenium and Vitamin E (Kim et al., 2003; Hu et al., 2017).

Sub-Saharan Africa, with widespread poverty (a risk factor for micronutrient deficiency) and the highest incidence of cervical cancer worldwide, has not been able to implement a successful population based screening and treatment program for cervical cancer control due to unavailability of the required resources and a weak health system infrastructure. Similarly, there is a paucity of published data on the relationship between HPV infection, micronutrients such as selenium and the development of cervical cancer from Africa.

This study was therefore designed to evaluate the relationship between serum selenium levels in women with CIN and those with normal cervical cytology. In 
addition, it attempts to explore if any trend exists in the mean serum selenium values and worsening severity of CIN. The findings from this work can assist in the provision of evidence for a nutrient mediated program in the control of cervical cancer which will be comparatively cheaper and simpler to implement than existing standards of care.

\section{Materials and Methods}

This study was a case control study conducted at the Gynae-oncology unit, department of Obstetrics and Gynaecology, University of Benin Teaching Hospital., Nigeria from January 2015 to December 2015. The Hospital has a comprehensive cervical cancer control program which includes screening using pap smears, colposcopy, surgery and radiotherapy. It performs over 1,500 smears and 200 colposcopy evaluations annually. Approval for the study was obtained from the Ethics and Research Committee of the University of Benin Teaching Hospital.

The cases consisted of women referred to the Gynae-oncology Clinic with an abnormal cervical smear for colposcopy and directed cervical biopsy which confirmed CIN. The next consecutive woman seen at the clinic with a normal Cervical histology served as the control. Inclusion criteria included women $\leq 65$ years old attending the Gynae-oncology Clinic and who gave informed consent to participate in the study.

Pregnant women or persons with chronic illnesses such as cancer, hypertension, diabetes mellitus, chronic liver disease, renal failure and those on multivitamin supplementation were excluded. Interviewer administered data forms were used to collect information on socio-demographic and reproductive health characteristics. The research outcome of interest was the trend in the mean serum selenium values between the cases and controls and within the various sub-groups of CIN. Serum selenium level was determined with the use of the graphite Atomic Absorption Spectrophotometer (AAS) as described by Paschal and Kimberly (1986).

For case control studies with two independent samples and a continuous outcome variable, the minimum sample size of 86 was calculated for this study (Edwardes, 2001). It assumed a type I error $(\alpha)$ of $5 \%$, type II error $(\beta)$ of $20 \%$, a level of significance of 0.05 and case: control ratio of 1:1. Also, based on a previous work in Southern Nigeria (Babalola et al., 2003), the mean serum selenium level of $2.39( \pm .33 \mu \mathrm{mol} / \mathrm{L})$ was used with an effect size of $0.33 \mu \mathrm{mol} / \mathrm{L}$ considered significant for patients with CIN.

The generated data was analysed with the IBM Statistical Package for the Social Sciences, version 20.0 (SPSS Inc., Chicago IL USA) using inferential statistics. Categorical variables were expressed as absolute numbers with percentages and compared using the Chi square test or Fisher exact test where appropriate. The magnitude of observed differences between the variables was estimated using the odds ratio at $95 \%$ confidence interval. Continuous variables were presented as mean values with standard deviation and compared with the t test or Analysis of variance (ANOVA) test. The level of significance was set at $\mathrm{p}<0.05$.

\section{Results}

Ninety (90) participants were recruited for this study with 45 cases and 45 controls respectively. The distribution of CIN sub groups among cases were: $41.6 \%$ CIN I, 35.4\% CIN II, 22.8\% CIN III. Both groups had comparable socio-demographic characteristics. There was no significant difference between the cases and the controls in terms of their age group, social class and level of education. The overall mean serum selenium level for the study population was $55.90 \pm 19.14 \mu \mathrm{g} / \mathrm{L}$ (range 7.26-90.99 $\mu \mathrm{g} / \mathrm{L}$ ). The mean value for the cases (with CIN) was $52.32 \pm 20.46 \mu \mathrm{g} / \mathrm{L}$ while the value for the controls with normal cervix $(59.49 \pm 17.20 \mu \mathrm{g} / \mathrm{L})$. This difference was not statistically significant $\{\mathrm{p}-0.076,95 \%$

Table 1. Risk of CIN for Selected Socio-Demographic and Clinical Factors

\begin{tabular}{|c|c|c|c|}
\hline Factor & Case $[\mathrm{N}(\%)]$ & Control $[\mathrm{N}(\%)]$ & OR $(95 \% \mathrm{CI})$ \\
\hline \multicolumn{4}{|l|}{ Marital status } \\
\hline Married & $38(84.4)$ & $34(75.6)$ & 1 \\
\hline Widow & $4(8.9)$ & $6(13.3)$ & $2.00(0.37-10.92)$ \\
\hline Single & $3(6.7)$ & $5(11.1)$ & $1.20(0.13-11.05)$ \\
\hline \multicolumn{4}{|c|}{ Age at first marriage (years) } \\
\hline$<20$ & $7(16.7)$ & $5(12.5)$ & 1 \\
\hline $21-25$ & $14(33.3)$ & $15(37.5)$ & $0.66(0.16-2.63)$ \\
\hline $26+$ & $21(50.0)$ & $20(50.0)$ & $0.67(0.18-2.52)$ \\
\hline \multicolumn{4}{|l|}{ Parity } \\
\hline 0 & $7(15.6)$ & $7(15.6)$ & 1 \\
\hline $1-4$ & $23(51.1)$ & $30(66.7)$ & $1.00(0.20-4.96)$ \\
\hline $5+$ & $15(33.3)$ & $8(17.8)$ & $4.50(0.49-41.25)$ \\
\hline \multicolumn{4}{|c|}{ Age at first childbirth (years) } \\
\hline$<20$ & $5(13.2)$ & $5(13.2)$ & 1 \\
\hline $21-25$ & $13(34.2)$ & $15(39.5)$ & $0.76(0.16-3.66)$ \\
\hline $26+$ & $20(52.6)$ & $18(47.4)$ & $1.10(0.27-4.49)$ \\
\hline \multicolumn{4}{|l|}{ Coitarche } \\
\hline$>16$ & $39(86.7)$ & $40(90.9)$ & 1 \\
\hline$£ 16$ & $6(13.3)$ & $4(9.1)$ & $1.67(0.40-6.97)$ \\
\hline \multicolumn{4}{|c|}{ Life time sexual partners } \\
\hline 1 & $19(42.2)$ & $17(38.6)$ & 1 \\
\hline $2-4$ & $23(51.1)$ & $21(47.7)$ & $1.08(0.43-2.72)$ \\
\hline$>4$ & $3(6.7)$ & $6(13.6)$ & $0.52(0.12-2.20)$ \\
\hline \multicolumn{4}{|c|}{ Use of oral contraceptives } \\
\hline No & $36(80.0)$ & $35(77.8)$ & \\
\hline Yes & $9(20.0)$ & $10(22.2)$ & $1.17(0.39-3.47)$ \\
\hline \multicolumn{4}{|c|}{ Duration of OCP use } \\
\hline Non use & $36(80.0)$ & $35(77.8)$ & 1 \\
\hline$<2$ years & $7(15.6)$ & $7(15.6)$ & $0.92(0.30-2.99)$ \\
\hline $2-10$ years & $1(2.2)$ & $1(2.2)$ & \\
\hline $10+$ years & $1(2.2)$ & $2(4.4)$ & $0.49(0.43-5.58)$ \\
\hline \multicolumn{4}{|c|}{ Type of marriage } \\
\hline Monogamy & $36(85.7)$ & $38(95.0)$ & 1 \\
\hline Polygamy & $6(14.3)$ & $2(5.0)$ & $3.17(0.60-16.72)$ \\
\hline
\end{tabular}


Table 2. Comparison of Mean Serum Selenium Values between Controls and CIN Subgroups (Using ANOVA)

\begin{tabular}{lcc}
\hline Histological diagnosis & Number & $\begin{array}{c}\text { Mean selenium (SD) } \\
(\mathrm{mg} / \mathrm{L})\end{array}$ \\
\hline Normal & 45 & $59.38(17.39)$ \\
CIN I & 18 & $53.43(17.79)$ \\
CIN II & 17 & $59.52(17.06)$ \\
CIN III & 11 & $40.48(24.67)$ \\
\hline
\end{tabular}

Test for trend, 0.025 ; P value, 0.021

\section{CI $(-15.08-0.76)\}$.}

In Table 1, the risk of CIN was compared to selected reproductive health characteristics of the study population. Although the observed differences between both groups were not statistically significant, widows were twice as likely to have CIN compared to married women. Respondents who reported early sexual debut ( $<16$ years) had a 2 fold risk for developing CIN compared to those who did not while women in polygamous marriages were three times more likely to have CIN. Grand multiparous women (Para 5 and above) had a 5 fold increased risk of CIN compared to nulliparous women.

In Table 2, there was a statistically significant difference in the mean value of serum selenium observed between the controls and histological subgroups within the cases (CIN I, CIN II, and CIN III) using the ANOVA test $(p=0.021)$. Post Hoc tests still showed a statistically significant linear trend for serum selenium to decrease with increasing grade of CIN ( $p$ value $=0.025$ ) and a statistically significant difference between controls (with normal cervix) and CIN III ( $\mathrm{p}$ value $=0.016$ ).

\section{Discussion}

The results from this study shows that overall mean value of the serum selenium level reported is comparable to similar studies from this region of Nigeria (Babalola et al., 2003; Arinola and Charles-Davies, 2008; Nwagha et al., 2011; Karaye et al., 2015). Although it is lower than values reported from Asia and Europe, this may be explained by the fact that dietary and geographic factors (soil type) have been documented to influence levels of selenium in human serum (Thompson et al., 2002; Coombs, 2001; Stoffaneler and Morse, 2015). Also, although the cases had lower average selenium levels than the control group, this difference was not statistically significant. This implies an acceptance of the null hypothesis for this study. It is noteworthy that the case group in this study consisted of both low grade CIN 1 (majority of whom are able to ultimately clear their HPV infection) and high grade CIN 2 and CIN 3 who have a comparatively much high risk for progression of HPV infection to cervical cancer. Therefore, it is possible that this combination may have blunted the magnitude and significance of the observed difference in the selenium levels between both groups.

A systematic review with meta-analyses on persistent HPV infection and cervical neoplasia reported that HPV persistence was strongly and consistently associated with
Serum Selenium levels and Cervical Intraepithelial Neoplasia

CIN2 and CIN 3 (Koshiol et al., 2008). It reported that this association increased with increasing severity of the grade of CIN. It therefore suggested that, mild disease (CIN1) may represents currently active but mainly transient HPV infection that will ultimately regress due to the actions of other cofactors to HPV infection.

In our study, we noted a trend for a progressive decline in the mean serum selenium levels from the control group to increasing severity of CIN. This trend reached statistical significance between the control group and CIN 3. It is consistent with the results from the meta-analyses earlier highlighted. Also, a recent meta-analysis on the relationship between Selenium levels and cervical cancer using pooled estimates reported serum selenium levels in cervical cancer cases were also significantly lower than in controls (He et al., 2017). From an epidemiological standpoint, the low selenium levels in CIN and Cervical cancer cases suggests that women with high grade CIN disease may represent a distinct group from those with low grade disease. It is possible that they may have peculiar attributes that puts them at increased risk of cervical cancer from persistent HPV infection. The significantly reduced serum selenium levels in these women compared to controls may represent an impairment in their anti-oxidant pathway that has been shown to have a protective effect on the development of human cancers by mopping up injurious free radicals (Cunzhi et al., 2003; Kim et al., 2003).

There are limitations to the results from this study. The sample size was powered to detect significant differences between women with normal cervices and those with CIN. It was not powered to detect differences between sub groups of CIN and the control group. Also, although an important micronutrient in oxidative metabolism in humans, there are other important micro nutrients such as Vitamin A, Vitamin E, Copper and Zinc that help in oxidative metabolism in the body which were not investigated in this study. However, our results remain valid because selenium is an important component of the most important glutathione antioxidant system in humans.

Although epidemiological evidence currently suggests that there may be a possible protective effect of anti-oxidant nutrients in HPV persistence and the risk of developing of cervical cancer, the exact mechanism how this occurs isn't quite clear. The result from this work suggests that the critical population of interest in the natural history of HPV infection and progression to cervical cancer may be among persons with high grade CIN disease. Further well designed and adequately powered research is required to effectively explore this observation.

It is our conclusion that the serum selenium levels differ significantly between women with normal cervices and those with high grade CIN 3. This difference may be a pointer to the existence of other biomarkers especially antioxidant micronutrients that may be important cofactors that modulate the progression of HPV infection to CIN and invasive cervical cancer. If so, it presents an opportunity to use a cost effective and simple intervention like micronutrient supplementation and dietary advice in the control and prevention of cervical cancer especially in 
sub Saharan Africa which has been unable to replicate the successes recorded in developed countries using conventional screening techniques.

\section{Acknowledgements}

This project was funded by a Grant from the Tertiary Education Trust Fund (TETFUND) in Nigeria and approved by the institutional Health and Research Ethics Committee. We declare no conflict of interest.

\section{References}

Arinola OG, Charles-Davies MA (2008). Micronutrient levels in the plasma of Nigerian females with breast cancer. Afr $J$ Biotech, 7, 1620-23.

Babalola O, Anetor J, Adeniyi F (2003). Assessment of selenium status of healthy adults in South Western Nigeria. ASSET Series, 3, 111-20

Chih HJ, Lee AH, Colville L, Binns CW, Xu D (2013). A review of dietary prevention of human papillomavirus-related infection of the cervix and cervical intraepithelial neoplasia. Nutr Cancer, 65, 317-28.

Combs GF (2001). Selenium in global food systems. Br J Nutr, 85, 517-47.

Cunzhi H, Jiexen J, Xianwen Z, et al (2003). Serum and tissue levels of six trace elements and copper/zinc ratio in patients with cervical cancer and uterine myoma. Bio Trace Elem Res, 94, 113-22.

Edwardes MD (2001). Sample size requirements for case-control study designs. BMC Med Res Methodol, 1, 11.

Ferlay J, Soerjomataram I, Dikshit R et al (2015). Cancer incidence and mortality worldwide: Sources, methods and major patterns in GLOBOCAN 2012. Int J Cancer, 136, 359-86.

Franceschi S, Vaccarella S (2015). Beral's 1974 paper: A step towards universal prevention of cervical cancer. Cancer Epidemiol, 39, 1152-6.

Franco EL, Schecht NF, Saslow D (2003). The epidemiology of cervical cancer. Cancer J, 9, 348-59.

He D, Wang Z, Huang C, Fang X, Chen D (2017). Serum selenium levels and cervical cancer: Systematic review and meta-analysis. Biol Trace Elem Res, 179, 195-202.

Hu X, Li S, Zhou L, Zhao M, Zhu X (2017) Effect of vitamin E supplementation on uterine cervical neoplasm: A meta-analysis of case-control studies. PLoS One, 12, $\mathrm{e} 0183395$

Jia WL, Ding L, Ren ZY, et al (2016). Effect of both folic acid,p16 protein expression and their interaction on progression of cervical cancerization. Zhongua Liu Xing Bing Xue Za Zhi, 37, 1647-52.

Karaye KM, Yahaya IA, Lindmark K, Henein MY (2015). Serum selenium and ceruloplasmin in Nigerians with peripartum cardiomyopathy. Int J Mol Sci, 16, 7644-54.

Kim SY, Kim JW, Ko YS, et al (2003). Changes in lipid peroxidation and antioxidant trace elements in serum of women with cervical intraepithelial neoplasia and invasive cancer. Nutr Cancer, 47, 126-30

Koshiol J, Lindsay L, Pimenta JM, et al (2008). Persistent human papillomavirus infection and cervical neoplasia: A systematic review and meta-analysis. Am J Epidemiol, 168, 123-37.

Ma W, Melief CJ, van der Burg SH (2017). Control of immune escaped human papilloma virus is regained after therapeutic vaccination. Curr Opin Virol, 23, 16-22

Muwonge R, Ngo L, Ngoma T, et al (2016). Socio-demographic and reproductive determinants of cervical neoplasia in seven sub-Sahara African countries. Cancer Causes Control, 27, 1437-46.

Nwagha UI, Ogbodo SO, Nwogu-Ikojo EE, et al (2011). Copper and selenium status of healthy pregnant women in Enugu, southeastern Nigeria. Nig J Clin Pract, 4, 408-12.

Ozgu E, Yilmaz N, Baser E, et al (2016). Could 25-OH vitamin D deficiency be a reason for HPV infection persistence in cervical premalignant lesions?. J Exp Ther Oncol, 11, 177-80.

Pascal DC, Kimberly MM (1986). Automated direct determination of selenium in serum by electrothermal atomic absorption spectroscopy. At Spectrosc, 7, 75-8.

Stoffaneller R, Morse NL (2015). A review of dietary selenium intake and selenium status in Europe and the Middle East. Nutrients, 7, 1494-1537.

Thompson FE, Patterson BH, Weinstein SJ, et al (2002). Serum selenium and the risk of cervical cancer among women in the United States. Cancer Causes Control, 13, 517-26.

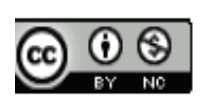

This work is licensed under a Creative Commons AttributionNon Commercial 4.0 International License. 